\title{
Determinação da resistência antimicrobiana associada em isolados clínicos de Staphylococcus aureus e Pseudomonas aeruginosa em um hospital público de Goiânia, Estado de Goiás
}

\author{
Determination of associated antimicrobial resistance in clinical isolates of \\ Staphylococcus aureus and Pseudomonas aeruginosa in a \\ public hospital in Goiânia, State of Goiás
}

\author{
Cláudia Castelo Branco Artiaga Kobayashi ${ }^{1,2}$, Geraldo Sadoyama ${ }^{1}$ \\ e José Daniel Gonçalves Vieira ${ }^{1}$
}

\begin{abstract}
RESUMO
Este estudo avaliou a resistência antimicrobiana associada de Pseudomonas aeruginosa e Staphylococcus aureus a um agente antimicrobiano com outras drogas. A resistência antimicrobiana associada foi calculada através do risco relativo. Houve uma relação óbvia entre resistência à oxacilina e a outros agentes antimicrobianos entre os isolados de Staphylococcus aureus resistentes à oxacilina (68,5\%) superior a 32\%, com exceção da linezolida (6,7\%). Resistência associada pronunciada entre drogas foi observada para isolados de Pseudomonas aeruginosa, particularmente entre ciprofloxacina e os carbapenens (59,6\% a 60,7\%), entre aminoglicosídeos e carbapenens (66,3\% a 67,7\%) e os demais $\beta$-lactâmicos $(52,3 \%$ a 85,8\%). 0 presente trabalho enfatiza a importância da cultura diagnóstica e do teste de suscetibilidade na seleção de um correto agente antimicrobiano com relação ao impacto clínico no aumento da multirresistência e na seleção de resistência antimicrobiana associada.
\end{abstract}

Palavras-chaves: Staphylococcus aureus. Pseudomonas aeruginosa. Resistência antimicrobiana associada.

\section{ABSTRACT}

This study evaluated the associated antimicrobial resistance of Pseudomonas aeruginosa and Staphylococcus aureus in relation to an antimicrobial agent with other drugs. The associated antimicrobial resistance was calculated by means of the relative risk. There was an obvious relationship between oxacillin resistance and resistance to other antimicrobial agents among isolates of oxacillin-resistant Staphylococcus aureus (68.5\%), greater than $32 \%$, except for linezolid (6.7\%). Pronounced associated resistance among drugs was observed for Pseudomonas aeruginosa isolates, particularly for ciprofloxacin and carbapenems (59.6\% to 60.7\%) and for aminoglycosides and carbapenems (66.3\% to $67.7 \%$ ) and other $\beta$-lactam antibiotics (52.3\% to 85.8\%). The present study emphasizes the importance of diagnostic cultures and susceptibility testing for selecting the correct antimicrobial agent, with regard to the clinical impact of increased multiresistance and selection of associated antimicrobial resistance.

Key-words: Staphylococcus aureus. Pseudomonas aeruginosa. Associated antimicrobial resistance.

Tem sido grande o impacto clínico e econômico em decorrência da presença de microrganismos resistentes, particularmente no ambiente hospitalar ${ }^{5}$, citando-se Staphylococcus aureus e Pseudomonas aeruginosa como os agentes multirresistentes mais comuns de infecção nosocomial ${ }^{325}$. As infecções causadas por estes patógenos são particularmente um desafio clínico, uma vez que o primeiro apresenta alta capacidade de desenvolvimento de resistência devido maior adaptação sob pressão seletiva do uso intenso de antimicrobianos ${ }^{31}$ e 0 segundo é caracterizado pela suscetibilidade natural a um número limitado de agentes

1.Instituto de Patologia Tropical e Saúde Pública, Universidade Federal de Goiás, Goiânia, GO. 2.Hospital de Urgências de Goiânia, Goiânia, GO.

Endereço para correspondência: Dr. José Daniel Gonçalves Vieira. Rua 235 s/n. Laboratório de Bacteriologia Médica/sala 418/IPTSP/UFG, Setor Universitário, 74605-050 Goiânia, GO.

Tel: 5562 3209-6361

e-mail: jdgvieira62@yahoo.com.br

Recebido para publicação em 17/04/2009

Aceito em 10/08/2009 antimicrobianos e elevadas taxas de resistência adquirida a várias drogas disponíveis ${ }^{21}$. 0 uso indiscriminado e/ou inadequado de antimicrobianos pode favorecer a taxa de resistência à droga em questão ou à outra relacionada, dificultando o controle de microrganismos multirresistentes ${ }^{3}$. Dessa forma, a emergência de resistência a uma classe de antimicrobiano após exposição à outra diferente tem grande importância clínica ${ }^{2}$. As estratégias terapêuticas empíricas tem se tornado problemáticas em função da alta prevalência de Staphylococcus aureus resistentes à oxacilina (ORSA) e Pseudomonas aeruginosa multirresistente ${ }^{729}$, nos quais a resistência a um determinado agente tem sido associada à resistência aumentada a antimicrobianos relacionados (resistência cruzada) e totalmente não relacionados entre si (resistência associada) ${ }^{32}$. Logo, o conhecimento da taxa de resistência e associação local são críticos para prescrições terapêuticas apropriadas ou alternativas, uma vez que as diferenças nos níveis de cura clínica podem existir baseadas na adequação do antimicrobiano prescrito ${ }^{19}$. Devido à maior frequiência de isolamento de Staphylococcus aureus e Pseudomonas aeruginosa em um hospital público de 
Goiânia ${ }^{112}$, este trabalho teve o objetivo de verificar a associação da resistência desses relevantes patógenos nosocomiais a um agente antimicrobiano com outras drogas, com a finalidade de possibilitar um auxílio nos protocolos terapêuticos, permitindo um tratamento empírico alternativo mais adequado.

\section{MATERIAL E MÉTODOS}

Foi realizada uma análise retrospectiva de dados relacionados a testes de suscetibilidade antimicrobiana de isolados de Pseudomonas aeruginosa e Staphylococcus aureus, provenientes de amostras clínicas de pacientes internados em um hospital público de Goiânia, referência em urgência e emergência, no período compreendido entre janeiro de 2006 a dezembro de 2008.

A identificação inicial destes microrganismos foi realizada através de métodos morfológicos e bioquímicos convencionais, de acordo com o Manual de Microbiologia Clínica para o Controle de Infecção em Serviços de Saúde ${ }^{16}$. Todos os testes de suscetibilidade foram realizados através do método de disco-difusão, segundo normas padronizadas pelo documento M2-A8, suplementado pelo M100-S17 do National Committee for Clinical Laboratory Standards (NCCLS)/ Clinical and Laboratory Standard Institute (CLSI) ${ }^{420}$.

Todos os isolados foram categorizados para suscetibilidade aos agentes antimicrobianos: ceftazidima, cefepime e aztreonam $(30 \mu \mathrm{g})$, imipenem e meropenem $(10 \mu \mathrm{g})$, ciprofloxacina, levofloxacina e gatifloxacina $(5 \mu \mathrm{g})$, sulfametoxazol-trimetoprim $(1,25 / 23,75 \mu \mathrm{g})$, piperacilina-tazobactam $(100 / 10 \mu \mathrm{g})$, oxacilina $(1 \mu \mathrm{g})$, rifampicina $(5 \mu \mathrm{g})$, eritromicina $(15 \mu \mathrm{g})$, clindamicina $(2 \mu \mathrm{g})$, gentamicina $(10 \mu \mathrm{g})$, amicacina $(30 \mu \mathrm{g})$ e linezolida


com cepas padrão de Staphylococcus aureus ATCC 29213 e Pseudomonas aeruginosa ATCC $27853^{4}$.

A multirresistência para Staphylococcus aureus foi definida como resistência à oxacilina e três ou mais dos seguintes agentes: eritromicina, clindamicina, rifampicina, gentamicina, tetraciclina e sulfametoxazol-trimetoprim ${ }^{10}$ e para Pseudomonas aeruginosa a resistência a pelo menos três dos seguintes antibióticos: amicacina, gentamicina, aztreonam, ceftazidima, cefepima, ciprofloxacina, imipenem, meropenem, piperacilina-tazobactam ou ticarcilina-clavulanato ${ }^{21}$.

Para cada espécie, a resistência antimicrobiana a uma droga foi calculada na presença e ausência de resistência a outra droga analisada. Resistência cruzada foi definida como a resistência aumentada a dois ou mais antimicrobianos dentro da mesma classe, enquanto a associada considerou-se a resistência aumentada a duas ou mais drogas de diferentes classes?.

A resistência associada foi quantificada calculando-se o RR (risco relativo) com diferenças significativas quando $p<0,05$, através do teste $\chi^{2}$ ou exato de Fisher, utilizando-se o software Epi Info v. 2000, CDC.

0 presente trabalho foi aprovado pelo Comitê de Ética em Pesquisa do Hospital de Urgências de Goiânia, atendendo a resolução 196/96, sob o protocolo CEP/HUGO/SES N $084 / 08$.

\section{RESULTADOS}

Do total de 1.960 bactérias isoladas, foram analisadas 1.239 cepas de Staphylococcus aureus e 721 de Pseudomonas aeruginosa. Estes microrganismos foram provenientes dos espécimes clínicos: secreção de feridas de sítio cirúrgico, intraabdominais, escaras, abscessos, drenos (1.042), urina (358), ponta de cateter (183), sangue (155), líquidos corporais (55), fragmentos ósseos (81), secreções do trato respiratório (56), tecido (27) e corpo estranho (3).

A resistência individual verificada em Staphylococcus aureus foi mais elevada à penicilina $(97,6 \%)$, eritromicina $(70,4 \%)$, clindamicina $(68 \%)$, oxacilina $(68,5 \%)$, gentamicina $(62,9 \%)$, sulfametoxazol-trimetoprim $(53,5 \%)$ e à ciprofloxacina $(53,1 \%)$. Por outro lado, a menor taxa foi verificada para gatifloxacina $(19,8 \%)$, levofloxacina $(23,7 \%)$ e rifampicina $(24,8 \%)$, sendo que taxa de suscetibilidade elevada foi verificada à linezolida $(99,1 \%)$. Ao observar o perfil de resistência múltipla de Staphylococcus aureus, 815 (65,8\%) isolados foram multirresistentes, sendo a maioria resistente a 5 (266) e 6 (221) antimicrobianos.

Ao analisarmos a não suscetibilidade antimicrobiana de Staphylococcus aureus na ausência e na presença de resistência a outras drogas, verificou-se que a resistência a um agente foi mais associada com a resistência aumentada a outras drogas relacionadas. No entanto, também foi evidente entre antimicrobianos totalmente não relacionados entre si. A taxa e o risco relativo de resistência com os perfis de resistência cruzada e associada dos isolados de Staphylococcus aureus tanto sensíveis como resistentes a outros agentes antimicrobianos são mostrados na Tabela 1.

A resistência à clindamicina ocorreu em 94,7\% dos isolados de Staphylococcus aureus resistentes à eritromicina, mas em 3,1\% dos sensíveis; e a resistência à eritromicina (99\%) também foi mais alta em isolados resistentes à clindamicina que entre os isolados sensíveis $(15,4 \%)$. Da mesma forma, a resistência à oxacilina foi verificada em 94,8\% dos isolados de Staphylococcus aureus resistentes à ciprofloxacina comparado com 7,9\% de Staphylococcus aureus sensível. Verificou-se que mais de 50\% dos isolados de Staphylococcus aureus foram resistentes à sulfametoxazol-trimetoprim e que a resistência a esta droga na presença de resistência a todos os outros agentes antimicrobianos foi superior a $80 \%$ dos isolados, exceto à penicilina $(65,4 \%)$. A despeito da menor taxa de resistência à linezolida $(0,9 \%)$, essa foi observada em 4,4\% a 18,5\% dos isolados de Staphylococcus aureus resistentes a todas as drogas testadas, com um risco de 4 a 7 vezes maior de ocorrer entre os isolados resistentes à oxacilina, gatifloxacina, sulfametoxazol-trimetoprim e rifampicina do que nos sensíveis.

Os resultados encontrados nesta pesquisa demonstraram também uma associação óbvia entre isolados de ORSA e resistência a outros agentes antimicrobianos como eritromicina $(99,7 \%$, RR:4,1), clindamicina (99,2\%, RR:7,3), ciprofloxacina (94,8\%, RR:12,0), levofloxacina (72,1\%, RR:11,9), sulfametoxazoltrimetoprim (87,8\%, RR:8,3), gentamicina $(95,2 \%$, RR:11,1), 
rifampicina (40,7\%, RR:23,9) e gatifloxacina (32,7\%, RR:22), incluindo à linezolida (6,7\%, RR:4,2), sendo a maioria dos isolados de ORSA considerada multirresistente (815/849). Esta associação de resistência foi mais significativa $(p=0,000)$ nos isolados resistentes à oxacilina que nos sensíveis, exceto à linezolida $(6,7 \%, \mathrm{RR}: 4,2, p=0,12)$.

Com relação aos isolados de Pseudomonas aeruginosa, os compostos mais ativos foram piperacilina-tazobactam (84,6\%) e levofloxacina (72,3\%), seguidos pelos carbapenens imipenem

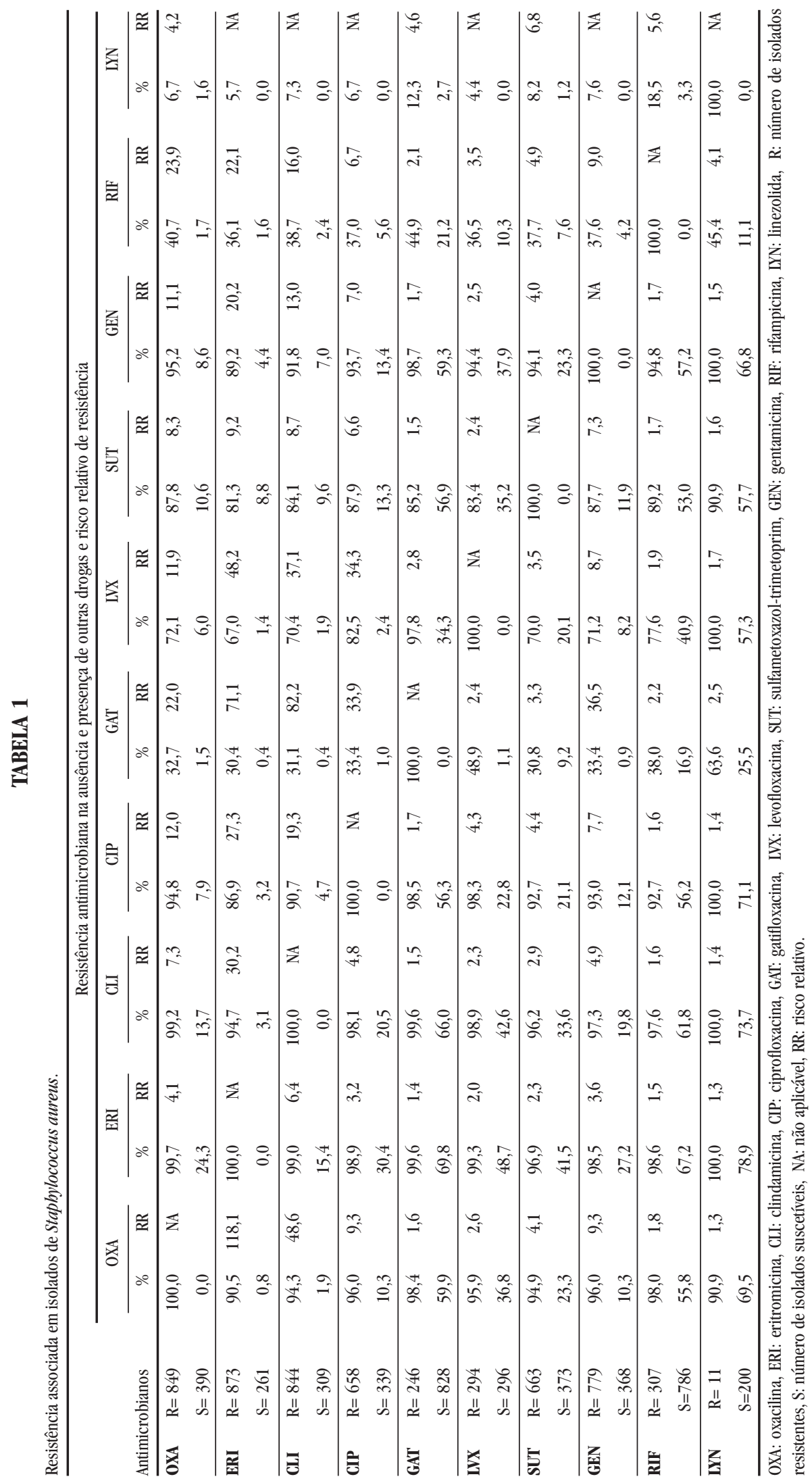


(69,1\%) e meropenem (67,8\%). A resistência aos antimicrobianos ciprofloxacina, gentamicina, cefalosporinas antipseudomonas e aztreonam foi bastante elevada, superior a $40 \%$.

Concernente a resistência associada, tanto a resistência aos agentes estruturalmente relacionados (cefalosporinas de $3^{\text {a }}$ geração, monobactâmico e carbapenens) como a resistência aos agentes não relacionados (quinolonas e aminoglicosídeos) apresentaram risco relativo estatisticamente significante $(\phi=0,000)$. No entanto, apesar de ser mais comum a presença de resistência às drogas relacionadas, verificou-se um maior risco de resistência associada entre drogas não relacionadas. A Tabela 2 mostra a resistência associada entre

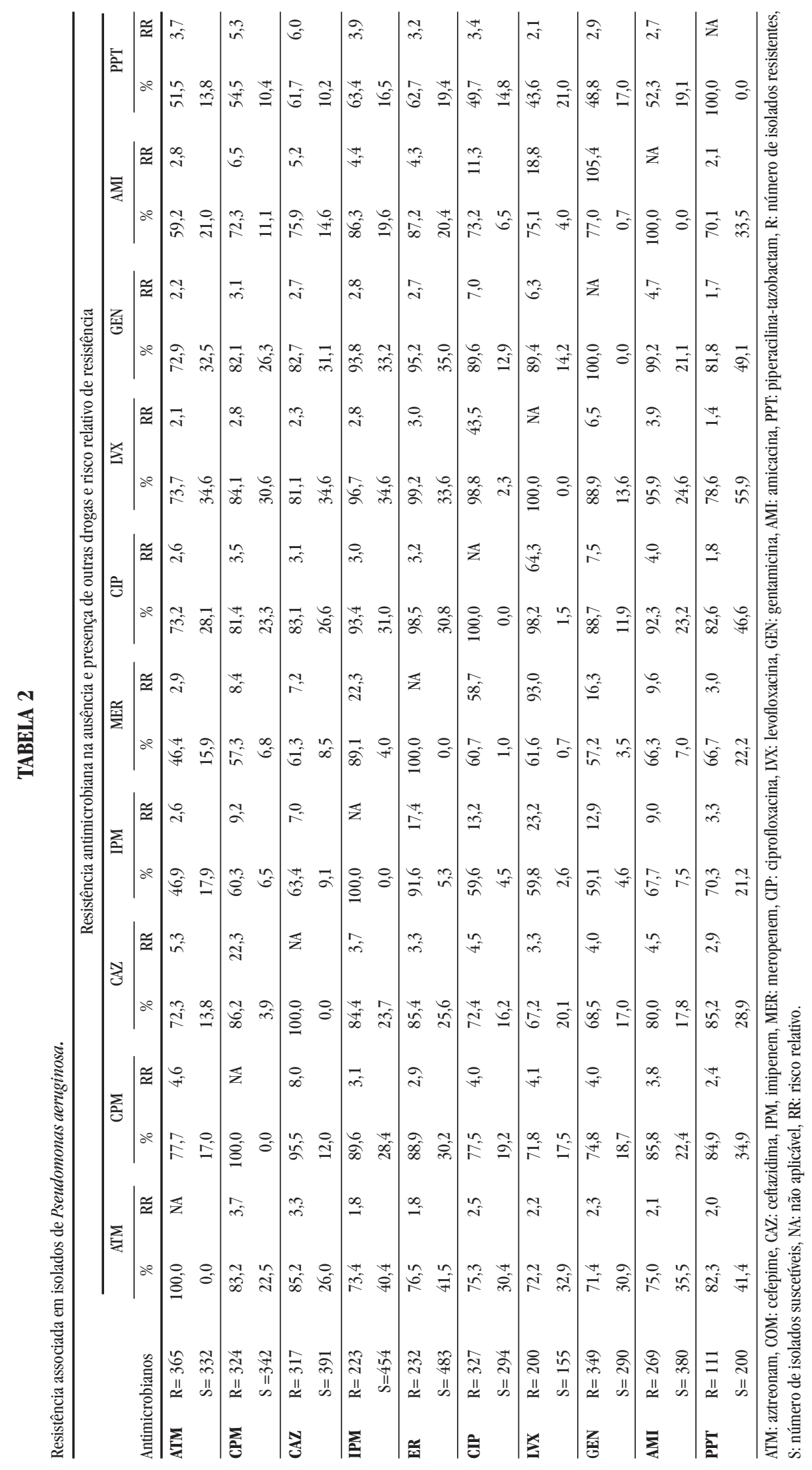


os isolados de Pseudomonas aeruginosa frente às drogas analisadas e o risco relativo de resistência.

A resistência à meropenem foi 22 vezes mais comum em isolados de Pseudomonas aeruginosa resistentes à imipenem, mas quase 60 vezes em isolados resistentes à ciprofloxacina e 16 vezes em isolados resistentes à gentamicina. Da mesma forma, ao se analisar a resistência aos aminoglicosídeos verificou-se maior risco de resistência associada às quinolonas (88,7-95,9\%, RR: 3,9-7,5). Nosso estudo também demonstrou que a resistência à ciprofloxacina foi associada a um aumento da resistência aos carbapenens entre 59,6\% a 60,7\% (RR: 13,2-58,7). Além disso, a resistência associada da ciprofloxacina com os demais $\beta$-lactâmicos ficou entre 49,7\% e 77,5\% (RR: 2,5-4,5). A taxa de resistência associada da amicacina com os carbapenens variou de 66,3\% a 67,7\% (RR: 9-9,6) e foi ainda mais alta quando associada ao demais $\beta$-lactâmicos (52,3\% a 85,8\%, RR: 2,7-4,5).

\section{DISCUSSÃO}

Altas taxas de resistência individual entre os isolados de Staphylococcus aureus às drogas antiestafilocócicas foram verificadas neste estudo, o que limita a utilidade destes antimicrobianos como terapia empírica em suspeita de infecção por ORSA. Além disso, o isolamento freqüente e a resistência múltipla individual de ORSA chamam a atenção para um problema endêmico na instituição estudada. Outros estudos, também, verificaram taxas elevadas de $\mathrm{ORSA}^{10} 14$

A ocorrência da resistência à linezolida em estabelecimentos clínicos tem sido considerada um fenômeno raro ${ }^{13}$, sendo mais relatada em pacientes debilitados sob longa terapia com este agente e em uso de dispositivos infectados não removidos ${ }^{18}$. Apesar da menor freqüência de resistência de Staphylococcus aureus à linezolida, o achado de resistência associada a este antimicrobiano é preocupante, visto que esta droga tem sido considerada uma das últimas opções terapêuticas para infecções graves causadas por isolados de ORSA, frequentemente multirresistentes ${ }^{7}$. Desta forma, estes resultados sugerem o uso mais restrito da linezolida, a fim de garantir o máximo da sua atividade em infecções mais graves.

Como detectado nesta investigação, Wimmerstedt e Kahlmeter ${ }^{32}$ também observaram uma taxa de resistência associada elevada à clindamicina $(58,3 \%)$ e à eritromicina $(97,2 \%)$ entre os isolados resistentes à contrapartida de ambas. A resistência elevada à eritromicina pode ser devido ao maior uso desta droga no tratamento de infecções humanas causadas por Staphylococcus aureus $^{8}$.

No presente estudo, observou-se uma elevada associação da resistência entre sulfametoxazol-trimetoprim e os demais agentes antimicrobianos investigados (superior a 80\%), assim como entre gentamicina (superior a 89\%) e as demais drogas. Apesar de relatos demonstrando ser o sulfametoxazol-trimetoprim um antimicrobiano oral apropriado para a terapia empírica de infecções por ORSA, particularmente de pele ou tecidos moles $^{28}$, a resposta clínica pode não ser satisfatória na instituição analisada. Da mesma forma, a alta taxa verificada de resistência à gentamicina sugere o uso de forma mais moderado da sua combinação com vancomicina para o tratamento de infecções graves por isolados de ORSA.

A associação da resistência à oxacilina com a resistência aumentada a outros antimicrobianos é condizente com a literatura atual. Sadoyama cols ${ }^{26}$, ao compararem os isolados nosocomiais de Staphylococcus aureus, observaram um porcentual de resistência elevada (>90\%) para eritromicina, clindamicina, gentamicina, ciprofloxacina, sulfametoxazol- trimetoprim e rifampicina entre os isolados ORSA. Kim cols ${ }^{10}$ verificaram uma maior resistência entre os isolados de ORSA para a eritromicina $(97,7 \%)$, gentamicina (95\%), clindamicina $(84,3 \%)$ e menor para a rifampicina (18\%) e sulfametoxazol-trimetoprim $(8,9 \%)$. 0 presente trabalho demonstrou porcentagens semelhantes, no entanto, a taxa de resistência à rifampicina (40,7\%) e sulfametoxazol-trimetoprim $(87,8 \%)$ nos isolados de ORSA foi mais alta. Isso demonstra a necessidade de maior cautela com relação ao uso empírico de ambos antimicrobianos, particularmente nos casos de uma opção terapêutica com melhor penetração tecidual que os glicopeptídeos (terapia principal para infecções sistêmicas por este patógeno), e de administração oral nos casos que requerem longo período, como na presença de material prostético ${ }^{10}$. Na instituição investigada, também foi observada uma associação elevada de resistência entre ORSA e às fluoroquinolonas, classe de antimicrobianos comumente prescritos no ambiente hospitalar. A exposição às fluoroquinolonas durante o tratamento é considerada um fator de risco significante para 0 isolamento de $\mathrm{ORSA}^{30}$. A associação entre fluoroquinolonas e outros antimicrobianos e ORSA atribuída exclusivamente à pressão seletiva favorece a aquisição e emergência de cepas resistentes às estes agentes ${ }^{30}$.

A resistência individual elevada em isolados de Pseudomonas aeruginosa à ciprofloxacina, aminoglicosídeos, cefalosporinas antipseudomonas e aztreonam foi mencionada por outros pesquisadores. Dados semelhantes foram verificados por Figueiredo $\operatorname{cols}^{6}$, ao determinarem uma taxa de sensibilidade variando entre 48,6\% e 59,8\% para os aminoglicosídeos, ciprofloxacina, cefepime e aztreonam. Estes pesquisadores observaram taxas de resistência aos carbapenens mais altas $(57,7$ a 58,2\% de suscetibilidade) e uma suscetibilidade de 66,1\% para piperacilina-tazobactam, inferior aos resultados encontrados neste trabalho, no qual foi demonstrada uma boa atividade deste antimicrobiano. As fluoroquinolonas e os aminoglicosídeos, drogas às quais a resistência foi elevada, tem se tornado um grande problema clínico, particularmente a ciprofloxacina, por apresentar maior potencial em induzir multirresistência bacteriana ${ }^{22}$. A baixa sensibilidade à ciprofloxacina tem sido associada com o aumento do seu uso a nível ambulatorial e hospitalar ${ }^{17}$. Além disso, essa quinolona tem sido identificada como um fator de risco para aquisição de Pseudomonas aeruginosa com suscetibilidade diminuída à mesma, à piperacilina, à ceftazidima e ao imipenem, em pacientes em unidade de terapia intensiva ${ }^{23}$.

A presença de resistência associada entre antimicrobianos não relacionados salienta que o desenvolvimento desta resistência ocorre primariamente entre bactérias já resistentes a um ou 
mais agentes antimicrobianos ${ }^{32} .0$ mesmo padrão de resistência associada em Pseudomonas aeruginosa, na qual foi evidente a resistência a uma droga na presença de resistência a outros agentes antimicrobianos, independente de pertencerem ou não a grupos relacionados, foi verificado por outros pesquisadores. Em Pseudomonas aeruginosa, Wimmerstedt e Kahlmeter ${ }^{32}$ observaram que a resistência entre drogas relacionadas, como à ceftazidima foi cerca de 7 vezes mais alta em isolados resistentes ao imipenem $(5,3 \%)$ que nos sensíveis $(0,8 \%)$; também evidenciaram uma resistência marcante entre drogas estruturalmente não relacionadas, como uma maior ocorrência de resistência à ciprofloxacina (5 a 10 vezes mais comum) em isolados resistentes a qualquer uma das drogas investigadas (ceftazidima, imipenem, gentamicina, piperacilina) que em isolados sensíveis. Paviani col $^{24}$ verificaram um percentual de resistência cruzada superior a $50 \%$ entre isolados resistentes às cefalosporinas e ao imipenem, sugerindo uma possível relação do aumento na resistência observada para estas drogas com elevadas taxas de consumo do carbapenem. 0 aumento do uso não criterioso de antimicrobianos de amplo espectro, particularmente $\beta$-lactâmicos, considerados grandes indutores de resistência ${ }^{15}$, tem sido relatado como um problema clínico bastante preocupante, já que envolve a busca constante de uma terapia empírica alternativa, dentre as poucas que restam. A seleção de resistência associada também foi observada por Alyassen $\operatorname{cols}^{2}$ ao demonstrarem que a exposição in vitro de Pseudomonas aeruginosa à ciprofloxacina seleciona isolados com não suscetibilidade tanto a esta droga como à cefepime, enquanto a exposição à cefepime seleciona para cefepime, mas não resistência à ciprofloxacina. Figueiredo $\operatorname{cols}^{6}$ mostraram taxas de resistência associada entre os isolados resistentes à ciprofloxacina e aos carbapenens (37\% para o imipenem e 38,1\% para o meropenem). Nosso estudo demonstrou um percentual mais elevado de resistência à ciprofloxacina associada a um aumento da resistência aos carbapenens $(59,6 \%$ a 60,7\%). Estes pesquisadores também apresentaram uma taxa de resistência associada da amicacina com os carbapenens de $32,4 \%$ a $33,3 \%$, inferior à encontrada na presente pesquisa. Shannon e French $^{27}$ observaram que os isolados resistentes à gentamicina foram cerca de 4 vezes mais prováveis de serem resistentes à ceftazidima ou aos carbapenens e, aproximadamente 8 vezes à ciprofloxacina, que os isolados de Psedomonas aeruginosa sensíveis.

Os resultados de resistência associada em Pseudomonas aeruginosa presumem a dificuldade de se estabelecer quais opções de associação de antimicrobianos para 0 tratamento de infecções graves causadas por este patógeno. Em função dos aminoglicosídeos e quinolonas, drogas comumente utilizadas como adjuvantes na terapêutica combinada com um $\beta$-lactâmico ${ }^{6}$ apresentarem percentuais de resistência associada com outros antibióticos bastante elevados, supõe-se que o potencial terapêutico dessa associação seja reduzido. Portanto, a resistência aos carbapenens, à ceftazidima e à cefepime (armas principais contra Pseudomonas aeruginosa), principalmente quando associada aos outros antimicrobianos, como aminoglicosídeos e quinolonas, representam um maior desafio para o tratamento ${ }^{29}$. Isso reflete no aumento da mortalidade e da permanência hospitalar e na necessidade maior de procedimentos diagnósticos e/ou terapêuticos ${ }^{1}$.
0 presente trabalho acentua a importância da seleção de um agente antimicrobiano individual com relação ao impacto clínico decorrente do aumento da multirresistência e da seleção de resistência cruzada e/ou associada. Epidemiologicamente, é necessário enfatizar 0 valor da identificação e determinação da suscetibilidade do microrganismo, não só para indicar a terapia empírica de primeira linha, mas como um auxílio ao clínico para a escolha de um agente antimicrobiano alternativo efetivo, caso haja falha no tratamento empírico primário.

\section{REFERÊNCIAS}

1. Aloush V, Navon-Venezia S, Seigman-Igra Y, Cabili S, Carmeli Y. Multidrug-resistant Pseudomonas aeruginosa: risk factors and clinical impact. Antimicrobial Agents and Chemotherapy 50: 43-48, 2006.

2. Alyaseen SA, Piper KE, Rouse MS, Steckelberg JM, Patel R. Selection of crossresistance following exposure of Pseudomonas aeruginosa clinical isolates to ciprofloxacin or cefepime. Antimicrobial Agents and Chemotherapy 49: 2543$2545,2005$.

3. Bernardes RC, Jorge AOC, Leão MVP. Sensibilidade à oxacilina, vancomicina e teicoplanina de Staphylococcus coagulase-positivos isolados de pacientes hospitalizados em São José dos Campos. Revista Biociências 10: 73-78, 2004.

4. Clinical and Laboratory Standard Institute. Performance Standards for Antimicrobial Susceptibility Testing; Seventeenth Informational Supplement. CLSI document M100-S17. Clinical and Laboratory Standards Institute 27: 1-182, 2007.

5. Engemann JJ, Carmeli Y, Cosgrove SE, Fowler VG, Bronstein MZ, Trivette SL, Briggs JP, Sexton DJ, Kaye KS. Adverse Clinical and Economic Outocomes Attributable to Methicillin Resistance among Patients with Staphylococcus aureus Surgical Site Infection. Clinical Infectious Diseases 36: 592-598, 2003.

6. Figueiredo EAP, Ramos H, Maciel MAV, Vilar MCM, Loureiro NG, Pereira RG. Pseudomonas aeruginosa: frequiência de resistência a múltiplos fármacos e resistência cruzada entre antimicrobianos no Recife/PE. Revista Brasileira de Terapia Intensiva 19: 421-427, 2007.

7. Fluit AC, Wielders CLC, Verhoef J, Schmitz FJ. Epidemiology and susceptibility of 3051 Staphylococcus aureus isolates from 25 University Hospitals participating in the European SENTRY study. Journal of Clinical Microbiology 39: 3727-3732, 2001.

8. Hernández NMR, López NF, Hernández MHE, Ferrer AF. Patrones de drogorresistencia de cepas de Staphylococcus aureus de origen clínico humano. Revista Cubana de Medicina Tropical 53: 53-58, 2001.

9. Kahlmeter G, Menday P. Cross resistance and associated resistance in 2478 Escherichi coli from the Pan-European ECO.SENS project surveying the antimicrobial susceptibility of pathogens from uncomplicated urinary tract infections. Journal of Antimicrobial Chemotherapy 52: 128-131, 2003.

10. Kim HB, Jang HC, Nam HJ, Lee YS, Kim BS, Park WB, Lee KD, Choi Y J, Park SW, Oh MD, Kim EC, Choe KW. In vitro activities of 28 antimicrobial agents against Staphylococcus aureus isolated from tertiary-care hospitals in Korea: a nationwide survey. Antimicrobial Agents and Chemotherapy 48: 1124-1127, 2004 .

11. Kobayashi CCBA, Sousa ED, Sadoyama G, Vieira JDG. Frequência de cocos grampositivos multirresistentes isolados em amostras clínicas de pacientes internados no Hospital de Urgências de Goiânia (HUGO), Go-Brasil. In: Resumos do $1^{\circ}$ Simpósio Internacional de Microbiologia Clínica, Gramado, p.658, 2008a.

12. Kobayashi CCBA, Sousa ED, Sadoyama G, Vieira JDG. Frequência de bacilos gram-negativos multirresistentes isolados de pacientes internados no Hospital de Urgências de Goiânia (Hugo), Go - Brasil. In: Resumos do $1^{\circ}$ Simpósio Internacional de Microbiologia Clínica, Gramado, p. 654, 2008b.

13. Kola A, Kirschner P, Gohrbandt B, Chaberny IF, Mattner F, Struber M, Gastmeier P, Suerbaum S. An infection with linezolid-resistant $S$. aureus in a patient with left ventricular assit system. Scandinavian Journal of Infectious Diseases 39: 463-465, 2007. 
14. Leão LSNO, Passos XS, Reis C, Valadão LMA, Silva MRR, Pimenta FC. Fenotipagem de bactérias isoladas em hemoculturas de pacientes críticos. Revista da Sociedade Brasileira de Medicina Tropical 40: 537-540, 2007.

15. Lepper PM, Grusa E, Reichl H, Hogel J, Trautmann M. Consumption of imipenem correlates with beta-lactam resistance in Pseudomonas aeruginosa. Antimicrobial Agents and Chemotherapy 46: 2920-2925, 2002.

16. Lewy CE, Von Nowakonski A, Mendes CMF, Oplustil C, Zoccoli CM, Marffei CM, Mamizuka EM, Cavassin ED, Rossi F, Mimica I, Petridis H, Mimica LMJ, Melhem MSC, Lopes MCG, Martino MDV, Lincopan N, Silva RAMS. Manual de Microbiologia Clínica para o Controle de Infecção em Serviços de Saúde. In: Módulo V. Detecção e identificação das bactérias de importância médica. Editora Agência Nacional de Vigilância Sanitária. $1^{a}$ edição, Ministério da Saúde. Brasília. p. 1-95, 2004.

17. Mcgowan JE. Resistance in nonfermenting gram-negative bactéria: multidrug resistance to the maximum. American Journal of Infection Control 34: S29-S37, 2006

18. Meka VG, Gold HS. Antimicrobial resistance to linezolid. Clinical Infectious Diseases 39: 1010-1015, 2004.

19. Miller LG, Quan C, Shay A, Mostafaie K, Bharadwa K, Tan N, Matayoshi K, Cronin J, Tan J, Tagudar G, Bayer AS. A prospective investigation of outcomes after hospital discharge for endemic, community-acquired methicillin-resistant and-susceptible Staphylococcus aureus skin infection. Clinical Infectious Diseases 44: 483-492, 2007.

20. National Committee for Clinical Laboratory Standards. Performance Standards for Antimicrobial Disk Susceptibility Tests; Approved Standard - Eighth edition. NCCLS document M2-A8. National Committee for Clinical Laboratory Standards, 23: p.1-58, 2003

21. Nouér SA, Nucci M, De-Oliveira MP, Pellegrino FLPC, Moreira BM. Risk factors for Acquisitilon of Multidrug-Resistant Pseudomonas aeruginosa Producing SPM Metallo-beta-lactamase. Antimicrobial Agents Chemotherapy 49: 3663-3667, 2005

22. Nseir S, Di Pompeo C, Soubrier S, Delour P, Lenci H, Roussel-Delvallez M, Onimus T, Saulnier F, Mathieu D, Durocher A. First-generation fluoroquinolone use and subsequent emergente of multiple drug-resistance bacteria in the intensive care unit. Critical Care Medicine 33: 283-289, 2005.
23. Paramythiotou E, Lucet JC, Timsit JF, Vanjak D, Paugam-Burtz C, Trouillet JL, Belloc S, Kassis N, Karabinis A, Andremont A. Acquisition of multidrug-resistant Pseudomonas aeruginosa in patients in intensive care units: role of antibiotics with antipseudomonal activity. Clinical Infectious Diseases 38: 670-677, 2004.

24. Paviani ER, Stadnik CB, Heinek I. Estudo da epidemiologia e perfil de sensibilidade da Pseudomonas aeruginosa. Infarma 15: 66-70, 2004.

25. Sader HS, Gales AC, Pfaller MA, Mendes RE, Zocolli C, Barth A, Jones RN. Pathogen frequency and resistance patterns in Brazilian hospitals: summary of results from three years of the SENTRY Antimicrobial Surveillance Program. Brazilian Journal of Infectious Diseases 5: 200-214, 2001.

26. Sadoyama G, Gontijo Filho PP. Risk factors for methicillin resistant and sensitive Staphylococcus aureus infection in a Brazilian University Hospital. The Brazilian Journal of Infectious Diseases 4: 135-143, 2000.

27. Shannon KP, French GL. Increasing resistance to antimicrobial agents of gramnegative organisms isolated at a London teaching hospital, 1995-2000. Journal of Antimicrobial Chemotherapy 53: 818-825, 2004

28. Szumowski JD, Cohen DE, Kanaya F, Maver KH. Treatment and outcomes of infections by methicillin-resistant Staphylococcus aureus at an ambulatory clinic. Antimicrobial Agents and Chemotherapy 51: 423-428, 2007.

29. Walsh TR, Toleman MA, Poirel L, Nordmann P. Metallo- $\beta$-lactamases: the quiet before the storm? Clinical Microbiology Reviews 18: 306-325, 2005.

30. Weber SG, Gold HS, Hooper DC, Karchmer AW, Carmeli Y. Fluoroquinolones and the risk for methicillin-resistant Staphylococcus aureus in hospitalized patients. Emerging Infectious Diseases 9: 1415-1422, 2003.

31. Weigel LM, Donlan RM, Shin DH, Jensen B, Clark NC, Mcdougal LK, Zhu W, Musser KA, Thompson J, Kohlerschmidt D, Dumas N, Limberger RJ, Patel JB High-level vancomycin-resistant Staphylococcus aureus isolates associated with a polymicrobial biofilm. Antimicrobial Agents and Chemotherapy 51: 231-238, 2007.

32. Wimmerstedt A, Kahlmeter G. Associated antimicrobial resistance in Escherichia coli, Pseudomonas aeruginosa, Staphylococcus aureus, Streptococcus pneumoniae and Streptococcus pyogenes. Clinical Microbiology and Infection 14: 315-321, 2008 